\title{
Efficiency of a New Combined Laser Therapy in Patients With Trophic Ulcers of Lower Extremities and Chronic Venous Insufficiency
}

\author{
Sergey Vladimirovich Moskvin ${ }^{1}$, Aleksandr Vladimirovich Geynitz ${ }^{2}$, Evgeniy Valer'yevich Askhadulin ${ }^{3^{*}}$ \\ ${ }^{1}$ State Scientific Center of Laser Medicine, Moscow, Russia \\ ${ }^{2}$ Reaviz Moscow Medical Institute, Moscow, Russia \\ ${ }^{3}$ Tula E.G. Lasarev Municipal Clinical Hospital №2, Russia
}

\section{*Correspondence to Sergey Moskvin, Doctor of Biological Sciences, Candidate of Engineering Sciences, Leading Researcher; State Scientific Center of Laser Medicine, Russia, Moscow. Tel: +79057701860 Fax: +7499 2505544; Email: 7652612@mail.ru}

Published online 27 June 2017

\begin{abstract}
Introduction: Treating patients with trophic ulcers (TUs) of venous etiology (VE) is a complicated socio-medical and economic problem. One of the ways to increase treatment efficiency can be a complex therapy, including combined laser action.

Methods: The objective of this study was a comparative evaluation of the efficiency of traditional treatment methods in patients with chronic venous diseases of C6 class, and combined laser therapy (LT) according to a new method by LASMIK ${ }^{\circledR}$ device. External exposure was conducted on the 1-4 affected area during one session for 2 minutes per zone (pulsed mode, light pulse duration of 100-130 ns, wavelength $635 \mathrm{~nm}$, by a matrix emitter consisting of eight laser diodes with a surface area of $8 \mathrm{~cm}^{2}$, at a distance of up to $7 \mathrm{~cm}$, pulsed power $40 \mathrm{~W}$ ). Intravenous laser blood illumination (ILBI) was conducted in continuous mode with a wavelength of 365-405 nm (UV-spectrum) and 520-525 nm (green spectrum) alternately, during 12 daily sessions, according to a special scheme. Results: The new method reduces time periods for wound surface cleansing from purulonecrotic masses, stimulates proliferation and epithelialization processes by 2-2.7 times, compared with the conventional method.

Conclusion: The high efficiency of a new combined LT has been demonstrated in the treatment of TU of VE in combination with traditional methods of patients' medical treatment.

Keywords: Trophic ulcer of venous etiology; Low level laser therapy; Venous Insufficiencies; Ulcer.
\end{abstract}

\section{Introduction}

Treating patients with trophic ulcer (TU) in lower extremities with venous etiology (VE) is a complicated socio-medical and economic problem. Prevalence of 'venous' TU in the adult working-age population of the Russian Federation is $1 \%-2 \%$, it occurs more often in female than in male, in $0.3 \%$ of TU of VE cases, the healing process takes a very long time with repeated recurrence. $^{1-4}$

Despite the advances of modern medicine, in particular, phlebology, the TU incidence rate has remained consistent over the past 20 years. There is a clear tendency towards the rejuvenation of this contingent of patients; it increases up to $4 \%-5 \%$ with age (in patients over 65 years old), and the radical elimination of the disease can be achieved only in every tenth patient. ${ }^{1}$ The problem is exacerbated by the presence of concomitant pathology. ${ }^{5}$

The main factors affecting patients' quality of life with venous TU include a pain syndrome $(80 \%$ of patients), resulting in insomnia (74\% of patients), significant everyday inconveniences associated with hygiene procedures ( $90 \%$ of patients) and a selection of comfortable shoes $(78 \%)$. Under the influence of these factors, psychological problems occur in the majority of patients. It was found that $26 \%$ of patients suffering from TU experience overwhelming fear to hurt their legs when playing games with children, $34 \%$ of them fear pets, another $27 \%$ cease communicating with their friends and colleagues. Only $3 \%$ TU patients have active sex life, most of them retaining normal potency. This has a negative impact on family relations and is a cause of divorce in $47 \%$ of cases. ${ }^{6}$

The cost of treating venous TU can vary within wide limits and depends on the technology used. In the countries of the European community, the closure of one TU as an outpatient procedure costs 800-1500 euros, and supporting anti-relapse treatment makes 100-150 euros per month. In the United States, for closure of venous TU

Please cite this article as follows: Moskvin SV, Geynitz AV, Askhadulin EV. Efficiency of a new combined laser therapy in patients with trophic ulcers of lower extremities and chronic venous insufficiency. J Lasers Med Sci. 2017;8(3):132-135. doi:10.15171/jlms.2017.24. 
within 3 months, direct medical costs reach US\$15002000. Prolongation of the treatment for a longer time results in increased cost up to US\$30000 or more. The total material damage, depending on the social status of the patient, can reach several hundred thousands of dollars. The annual costs on the health care system in the United States and Western Europe account for up to 2.5 billion euros. In Russia, the direct costs of treating one case of venous TU can reach 160-250 euros in the clinic, and exceed 325 euros in hospital. ${ }^{7-9}$

Great prevalence of TU of VE, peculiarities of the disease's progress and differences of wound healing process from other types of skin abnormalities, a tendency to relapse, poor efficiency of the existing therapies, the enormous economic damage caused by disability and frequent incapacitation of patients, as well as the need for additional care for elderly patients, dictate the necessity to find better, pathogenetically substantiated methods to treat this disease. ${ }^{10}$

Currently, the main treatment trends for patients with venous TU include (1) the correction of venous hemodynamics by surgery, sclerotherapy and elastic compression; (2) medication effects on venous tonus, micro-circulation and lymphatic drainage; (3) topical treatment, the nature of which is determined by the stage of the wound healing process, particularly applying physical factors, that is a subject of many works. ${ }^{11,12}$

There are numerous reports in literature referring to topical application of various kinds of low level laser therapy (LLLT), mainly in continuous mode of the red spectrum (633-635 nm) and in pulsed mode of infrared (IR) spectrum $(890-904 \mathrm{~nm})$ when treating TU. The abilities of LLLT non-invasive irradiation to restore the elasticity of the cell membranes, to normalize lymph and hemomicrocirculation in affected area, and to restore the regulating and sanogenetic functions of tissues, organs and the entire body by activating enzyme systems and metabolism, are very useful in the treatment of patients with venous TU. ${ }^{13-16}$

However, poor efficiency of the known methods of treating patients with TU necessitates the search for new ways and modes of laser therapy (LT) based on a comprehensive approach. Application of physical therapy, primarily with the use of LLLT, capable of influencing various stages in the pathogenesis of the disease, allows a high therapeutic effect in many cases, while ensuring systemic response of the patient's body with the proper exposure procedure.

LT methods for patients with TU of VE can be divided into 2 types: topical exposure and intravenous laser blood irradiation (ILBI). However, the available techniques are not sufficiently perfect, which is demonstrated by a rather large number of negative results of treatment or its minimal effect. This determined the relevance of the present study.

The objective of this study is to improve the results of outpatient treatment of patients with VE TU of lower extremities through the application of LLLT with different wavelengths.

\section{Methods}

This study was performed as a clinical trial (Clinical Trials. gov; Identifier: NCT03104985). During a longitudinal study, a group of patients was followed up in the period of complete or partial recovery. The analysis of the results of outpatient examination and treatment of 68 patients with venous TU, from 2013 to 2015, observed in the City Polyclinic of “Tula Municipal Clinical Hospital №2” was carried out.

The group of patient consisted of 50 (73.5\%) women and $18(26.5 \%)$ men. Patients' age varied from 39 to 78 years old. A mean age was $57.2 \pm 0.5$ years. Patients were examined as follows: general clinical methods of investigation (complete blood count, clinical urine analysis, biochemical blood assay, including glucose test, blood coagulation system test, culture and sensitivity tests of the ulcer defect surface) and instrumental investigations (ultrasonic Doppler examination [USDG], the measurement of the TU size with the calibration ruler).

Duration of the disease was above 5 years in 27 patients (39.7\%) and less than 5 years in 41 patients $(60.3 \%)$. There were $47(69.1 \%)$ patients with varicose disease and 21 (30.4\%) patients with post-thrombophlebitic disease.

International classification of lower extremity chronic venous disorders (CEAP) was used as a clinical classification of chronic venous insufficiency (CVI). In our survey, all patients had CVI Stage 6 (S6) in compliance with CEAP. ${ }^{17}$

TU were localized over the medial ankle in 40 (58.8\%) patients, over the lateral malleolus in $18(26.5 \%)$ patients, in the combination of contralateral surfaces in 10 (14.7\%). The disease recurrence was identified in 39 (57.3\%) patients when they visited the clinic. Ulcer defects sized below $5 \mathrm{~cm}^{2}$ in 32 (47\%) patients; from 5 to $10 \mathrm{~cm}^{2}$ in 28 (41.2\%) and from 10 to $25 \mathrm{~cm}^{2}$ in $8(11.8 \%)$.

Depending on the applied treatment method, patients were divided into 2 groups (Table 1 ).

Group 1 was represented by 34 (50\%) patients who underwent conventional therapy. It included:

- An elastic compression of lower extremities (elastic bandages or compression hosiery of class 2).

- Pharmacotherapy: to improve venous tonus the following drugs were used: Anavenol, Aescusan, Glyvenol; lymphatic drainage function was improved using drugs of the benzopyrone group, including Troxevasin and Venoruton. In order to eliminate microcirculation disturbances and regulate hemorheology, Trental, aspirin and Ticlid (ticlopidine) were used. Nonsteroidal anti-inflammatory drugs (Nimesil, OKI), various ointments containing heparin, corticosteroids, as well as nonsteroidal anti-inflammatory drugs were used to stop inflammation. Antibiotic therapy of ulcer defect was administered in accordance with the sensitivity after determining microflora by culture inoculation

- Topical treatment was administered according to the general surgical rules: in the presence of purulent discharge (phase I of the wound healing process), 
Table 1. Distribution of Patients Depending on the Treatment Method

\begin{tabular}{|c|c|c|c|}
\hline \multirow{2}{*}{ No. } & \multirow{2}{*}{ Treatment Methods } & \multicolumn{2}{|c|}{ Number of Patients } \\
\hline & & Abs. & $\%$ \\
\hline 1 & Conventional treatment & 34 & 50 \\
\hline 2 & $\begin{array}{l}\text { Conventional treatment }+ \text { combined LLLT, including external exposure with } 635 \mathrm{~nm} \text { wavelength }+ \text { ILBI } \\
\text { with } 365-405 \mathrm{~nm}(\text { UV-spectrum) and } 520-525 \mathrm{~nm} \text { (green spectrum) wavelength alternately, according } \\
\text { to the scheme }\end{array}$ & 34 & 50 \\
\hline Total & & 68 & 100.0 \\
\hline
\end{tabular}

bandaging with antiseptic solutions (1\% iodopiron solution, $0.1 \%$ chlorhexidine solution) and hydrophilic ointments (levosin, levomecol) were applied. In phases II and III, after ulcer cleansing, the treatment was aimed toward stimulating the granulation and marginal epithelization development. For this purpose, we used the preparations based on hyaluronic acid (Curiozin).

To carry out LT, the physiotherapeutic device LASMIK ${ }^{\circledR}$ was used (Registration Certificate in Russia № RZN 2015/2687 dated 25.05.2015). External exposure and ILBI were included in a daily complex of standard therapy. External exposure was conducted on the 1-4 affected area during one session for 2 minutes per zone in pulsed mode, light pulse duration of 100-130 ns, with wavelength $635 \mathrm{~nm}$, by a matrix emitter consisting of 8 laser diodes with a surface area of $8 \mathrm{~cm}^{2}$, at a distance of up to 7 $\mathrm{cm}$, with pulsed power of $40 \mathrm{~W}$. ILBI was conducted in continuous mode with wavelength between 365-405 $\mathrm{nm}$ (UV-spectrum) and 520-525 nm (green spectrum) alternately, during 12 daily treatment sessions according to the scheme:

- Session 1 - 365-405 nm, power 1-2 mW, exposure 2 minutes;

- Session 2 - 520-525 nm, power 1-2 mW, exposure 5 minutes;

- Session 3 - 365-405 nm, power 1-2 mW, exposure 2 minutes;

- Session 4 - 520-525 nm, power 1-2 mW, exposure 5 minutes;

- Session 5 - 365-405 nm, power 1-2 mW, exposure 2 minutes;

- Session 6 - 520-525 nm, power 1-2 mW, exposure 5 minutes;

- Session 7 - 365-405 nm, power 1-2 mW, exposure 2 minutes;

- Session 8 - 520-525 nm, power 1-2 mW, exposure 5 minutes;

- Session 9 - 365-405 nm, power 1-2 mW, exposure 2 minutes;

- Session 10 - 520-525 nm, power 1-2 mW, exposure 5 minutes;

- Session 11 - 365-405 nm, power 1-2 mW, exposure 2 minutes;

- Session 12 - 520-525 nm, power 1-2mW, exposure 5 minutes.

Patients in the control group were representative by age, sex, prevalence of lesions and the presence of comorbidities. Patients were distributed by random sampling; simulation of laser exposure was not performed.

\section{Results and Discussion}

Evaluation of clinical treatment results showed that a slight dynamics of changes in the clinical picture was identified in patients with venous TU of group 1, who received only conventional treatment. After 14 days of treatment, edema of shank reduced only in 9 (26.5\%) patients, and the pain stopped only in 7 (20.6\%) patients. In group 2, where in addition to conventional therapy, LLLT was performed according to the combined procedure, in contrast to the patients' group 1, who received only conventional treatment, pain reduction in the lower extremities and even much faster decrease in inflammatory phenomena in the TU area were noted in 5-7 days. Thus, surrounding tissue hyperemia persisted for 3-3.5 days, and infiltration around the edges of TUs remained for 4-4.5 days. Key indicators of wound healing process dynamics in patients with venous TU are given in Table 2.

As follows from the table data, in the group treated by conventional method, average TU cleansing time was 9.8 \pm 0.2 days, generation of granulation tissue was identified in $10.3 \pm 0.8$ days and a healing (50\% epithelialization) in $28.1 \pm 1.4$ days.

In the second group, the average time of cleansing from purulo-necrotic masses, granulation occurrence and ulcer epithelialization took, respectively: $6.4 \pm 0.4,7.1 \pm 0.6$ and $12.8 \pm 1.6$ days.

Compared to the patients of the first group, application of the developed technique for treating TU in patients with CVI of lower extremities promoted time reduction in ulcer cleansing by 1.5 , granulation occurrence by 1.4 times, wound healing ( $50 \%$ epithelialization) by 2.2 times. Conservative treatment was applied for patients with venous TU either as a preparation for surgical interventions aimed at correcting venous circulation of lower extremities, or as the main therapy, when there were contraindications to surgery or patients' categorical refusal of the operation.

The treatment results were evaluated after 6 months. Clinical dynamics were characterized by a more pronounced improvement in overall health, decrease in feeling of 'tiredness' in legs, reduced edema of shanks and feet in patients with venous TU in 6 months after treatment in group 2, which included external exposure with wavelength of $635 \mathrm{~nm}$ and ILBI with wavelength of 365-405 nm (UV-spectrum) and 520-525 nm (green 
Table 2. Key Indicators of Wound Healing Process Progression in the Surveyed Groups

\begin{tabular}{|c|c|c|c|c|}
\hline \multirow[b]{2}{*}{ Patient Groups } & \multicolumn{3}{|c|}{ Average Time (in days) } & \multirow{2}{*}{$\begin{array}{c}\text { Wound Healing }(\mathbf{1 0 0} \% \\
\text { Epithelialization) in } 6 \text { Months, } \\
\text { No. }(\%)\end{array}$} \\
\hline & Wound Surface Cleansing & Granulation Occurrence & $\begin{array}{c}\text { Wound Healing (50\% } \\
\text { Epithelialization) }\end{array}$ & \\
\hline Group $1(n=34)$ & $9.8 \pm 0.2$ & $10.3 \pm 0.8$ & $28.1 \pm 1.4$ & $11(32.3)$ \\
\hline Group $2(n=34)$ & $6.4 \pm 0.4^{\mathrm{a}}$ & $7.1 \pm 0.6^{a}$ & $12.8 \pm 1.6^{\mathrm{a}}$ & $28(82.3)$ \\
\hline
\end{tabular}

${ }^{a}$ Statistically significant difference of groups 1 and $2(P<0.05)$.

spectrum). The clinical picture of the first group corresponded to that prior to the therapy course.

In our opinion, the results of healing venous $\mathrm{TU}$ in groups are indicative. Thus, among patients receiving only conventional therapy, TU healing in 6 months was identified in $11(32.3 \%)$ patients. Ulcer epithelialization occurred in 28 (82.3\%) patients receiving conventional therapy and LT with external exposure and ILBI with wavelength of 365-405 nm (UVA-spectrum) and 520-525 nm (green spectrum).

Thus, it is shown that the proposed method allows to carry out more effective treatment of patients with TU of VE, reduce time of healing, and to stimulate quite pronounced and persistent adaptive physiological response, that prevents TU relapse development.

\section{Conclusion}

The new method of treating patients with TUs of VE using low level laser irradiation contributes to reduce time periods for wound surface cleansing from purulo-necrotic masses, stimulates proliferation and epithelialization processes by 2-2.7 times, compared with the conventional method, which allows improvement of treatment results for this group of patients, reducing time of ulcer healing, lowering the incidence of incapacitation and social adaptation costs.

\section{Conflict of Interests}

The authors declare no conflict of interest, financial or other exists.

\section{Ethical Considerations}

This study has been approved by ethical committee of Tula E.G. Lasarev Municipal Clinical Hospital №2, Russia.

\section{References}

1. Kruglova LS, Panina AN, Strelkovich TI. Trophic ulcers of venous genesis. Rossiyskiy Zhurnal Kozhnykh $i$ Venericheskikh Bolezney. 2014;17(1): 21-25. [Russian].

2. Obolensky VN, Rodoman GV, Nikitin VG, Karev MA. Trophic ulcers of lower extremities - the problem survey. Russkiy Meditsinskiy Zhurnal. 2009;17(25):1647-1663. [Russian].

3. Tolstykh PI, Tamrazova OB, Pavlenko VV, Kuleshov IY, Tolstykh MP. Dlitel'no ne zazhivayushchiye rany i yazvy (patogenez, klinika, lecheniye) (Long-term healing wounds and ulcers [pathogenesis, clinic, treatment]). Moscow: Dipak; 2009. [Russian].
4. Zhukov BN, Katorkin SE, Zhukov AA. Venous trophic ulcers of lower extremities. Diagnostics, treatment and medical rehabilitation [monograph]. Samara Meditsina;2012:236.

5. Kotelnikov GP, Losev II, Sizonenko YV, Katorkin SE. Peculiarities of diagnostics and tactics in treating patients with coexistent affection of musculoskeletal and venous systems of lower extremities. Novosti Khirurgi. 2013;21(3):42-53. [Russian].

6. Bogdanets LI. Strategy and tactics in treating trophic ulcers of venous genesis [Thesis]. Moscow; 2009. [Russian].

7. Kuznetsov NA, Rodoman GV, Nikitin VG, Karev MA, Shalayeva TI. The use of current bandages in the treatment of patients with venous trophic ulcers of the shin: clinical and economic. Khirurgiia NI Pirogov J. 2009;11:63-69. [Russian].

8. Rabe E, Pannier F. Societal costs of chronic venous disease in CEAP C4, C5, C6 disease. Phlebology. 2010;25(1):64-67. doi:10.1258/phleb.2010.010s09.

9. Lazarus G, Valle MF, Malas M, et al. Chronic venous leg ulcer treatment: Future research needs. Wound Repair Regen. 2014;22(1):34-42. doi: 10.1111/wrr.12102.

10. Karapetyan GE, Yakimov SV, Mikitin IL, Kochetova LV, Pakhomova LA. Modern method of maintaining trophic ulcers. Fundamentalnye Issledovaniya. 2013; 9(6):10161018. [Russian].

11. Moskvin SV. Revisiting the mechanisms of therapeutic action of low level laser irradiation. Vestnik Novykh Meditsinskikh Tekhnologiy. 2008; 15(1):166-172. [Russian].

12. Kalinina EV. Optimization of topical treatment of trophic ulcers of venous etiology [Thesis]. Moscow; 2010:30. [Russian].

13. Gavrilenko AV, Musaev MM. Laser techniques in combined therapy of patients with trophic ulcers of lower extremities of venous etiology. Khirurgiya. 2011;4:64-7. [Russian].

14. Katorkin SE, Zhukov AA, Melnikov MA, Kushnarchuk MY. Combined treatment of trophic ulcers during chronic venous diseases of lower extremities. Lasernaya Meditsina. 2015;19(3):23-28. [Russian].

15. Katorkin SE, Losev II, Sizonenko YV. Patients with venous and musculoskeletal disorders of the legs: functional and clinical methods for diagnosis and therapy. Novosti Khirurgii. 2014;26(1):55-61. doi:10.18484/2305-0047.2016.1.55. [Russian].

16. Patent 95262 RU. Persistent wound treatment device/ Zhukov BN, Melnikov MA, Kostuev VE, Zhukov AA. 2009143211/22; applied on 25.11.2009; published on 27.06.10, Bulletin No. 18; Priority of 25.11.2009.

17. Myshentsev PN, Zhukov BN, Katorkin SE, Yarovenko GV. The role of computer tomography in the evaluation of the lower extremities lymphedema stage. Novosti Khirurgii. 2011;19(5):74-77. [Russian]. 